\section{Modulating vaccine efficiency}

The live attenuated vaccine YF17D against yellow fever is one of the most successful vaccines yet developed. In the Journal of Clinical Investigation, Trautmann and colleagues examine baseline immunological parameters that can potentially modulate the efficacy of YF17D. Comparing two populations of YF17D vaccinees, one in Uganda and the other in Switzerland, the authors find higher replication of the attenuated virus in the Swiss cohort; however, this is associated with a more robust neutralizing antibody response and a higher frequency of YF17D-specific CD8 ${ }^{+} \mathrm{T}$ cells. The impaired YF17D response in the Ugandan group is associated with acrossthe-board baseline activation of innate and adaptive immunity, as well as some evidence of immunological exhaustion. The responses of memory $T$ cells and B cells in preimmunized Ugandan subjects also show diminished persistence. These results suggest that preexisting activation of the mmune system can impair the efficacy of YF17D and that boosting, which is not normally recommended by the guidelines of the World Health Organization, may be beneficial to such populations.

J. Clin. Invest. (9 June 2014) doi:10.1172/JCI75429

\section{Immune cell-brain cell messaging}

The brain and immune system communicate mainly by the exchange of soluble factors such as cytokines. In PLoS Biology, Momma and colleagues demonstrate that such communication also occurs through the delivery of exosomes. Using an experimental system in which Cre recombinase is conditionally expressed only in hematopoietic cells, the authors observe very low, steady-state delivery of Cre to Purkinje neurons in the absence of any apparent immunocyte-neuron fusion. This delivery is notably enhanced during inflammation. Cre protein itself is not released; instead, mRNA encoding Cre is packaged into exosomes, which are then released into the serum, especially in inflammatory conditions. There is no appreciable production of such exosomes by brain-resident cells of the immune system, such as microglia; instead, the exosomes are able to traverse the blood-brain barrier directly. Neurons taking up exosomes show altered expression of microRNA, which suggests that the exosomes influence the activity of these cells in an as-yet-unspecified manner. ZF

PLoS Biol. (3 June 2014) doi:10.1371/journal.pbio.1001874

\section{Coinfection wakes up latent viruses}

Herpesviruses can establish latent infections, but whether coinfection with other pathogens can reactivate the viruses remains unknown. In Science, Virgin and colleagues show that helminth coinfection stimulates the replication and production of herpesvirus. This effect requires signaling via cytokines that induce the transcription factor STAT6, such as interleukin 4 (IL-4) and IL-13, as well as suppression of interferon- $\gamma$ signaling. Many herpesviruses, including mouse $\gamma$-herpesvirus and human Kaposi's sacoma virus, seem to have evolved the ability to sense these competing signals that regulate the expression of a latentto-lytic 'switch' gene. Thus, herpesviruses are acutely tuned to the immunological status of their host and undergo latency or productive replication dependent on signaling by IL-4 or interferon- $\gamma$.

Science (26 June 2014) doi:10.1126/science.1254517

\section{Altering host to defeat tuberculosis}

Treating patients infected with Mycobacterium tuberculosis is becoming increasingly difficult because of the emergence of multidrug-resistant bacteria. In Nature, Mayer-Barber et al. show that it is possible to enhance the host-mediated control of M.tuberculosis by changing the innate immune response to infection. The production of type I interferons (IFN- $\alpha$ and IFN- $\beta$ ) exacerbates infection with $M$. tuberculosis by inhibiting protective responses induced by signaling via IL-1. Conversely, IL-1 triggers the production of antimycobacterial cytokines and nitric oxide and inhibits the expression of IFN- $\alpha$ and IFN- $\beta$. IFN- $\alpha-$ IFN- $\beta$ and IL- 1 elicit competing pathways of eicosanoid metabolism that lead to the synthesis of lipoxin or prostaglandin $\mathrm{E}_{2}\left(\mathrm{PGE}_{2}\right)$, respectively. The administration of either IL- 1 or $\mathrm{PGE}_{2}$ improves host control of infection with M. tuberculosis in mice. Retrospective analysis of cytokine profiles from patients with tuberculosis also shows that enhanced protection correlates with IL-1 and $\mathrm{PGE}_{2}$. Thus, it is possible to boost host control of $M$. tuberculosis by blocking the production of IFN- $\alpha$ and IFN- $\beta$ and enhancing IL-1 responses.

$L A D$ Nature (25 June 2014) doi:10.1038/nature13489

\section{Immunological function for TFEB}

The mammalian transcription factor TFEB and its Caenorhabditis elegans ortholog HLH-30 control the expression of genes encoding molecules involved in autophagy and lysosomal biogenesis in response to nutritional stress. In Immunity, Visvikis et al. show that HLH-30 drives the $C$. elegans transcriptional response to infection with Staphylococcus aureus. HLH-30-deficient worms show diminished survival after infection with $S$. aureus and other Grampositive and Gram-negative bacteria, as well as diminished survival in response to starvation and diminished longevity in nonpathogenic conditions. HLH-30 controls the induction of regulators of longevity, such as insulin-signaling genes, which do not affect host defense, and antimicrobial and autophagy regulators, which are required for host immune responses but have no effect on longevity. During infection of mouse macrophages with $S$. aureus, TFEB is required for the expression of proinflammatory cytokines, such as IL-6, IL-1 $\beta$ and TNF, and chemokines. Thus, the antimicrobial and autophagy roles of these functional homologs are evolutionarily conserved. Immunity 40, 896-909 (2014)

\section{LUBAC and the inflammasome}

The linear ubiquitin chain-assembly complex (LUBAC) mediates the linear ubiquitination of signaling mediators downstream of various Tolllike receptors to induce activation of the transcription factor NF- $\mathrm{KB}$ in fibroblasts and keratinocyes. In The Journal of Experimental Medicine, Rodgers et al. show that in mouse macrophages, the HOIL-1L subunit of LUBAC is required for linear ubiquitination of the adaptor ASC and for assembly of the NLRP3 inflammasome, but not for the activation of NF-кB. HOIL-1L-deficient bone marrow-derived macrophages have normal activation of NF- $\mathrm{\kappa B}$ but defective cleavage of pro-caspase-1 and defective formation of NLRP3-ASC complexes after stimulation of NLRP3. The HOIL-1L and HOIP units of LUBAC are both required for the linear, nondegradative ubiquitination of ASC after stimulation. HOIL-1L-deficient mice have diminished NLRP3-dependent production of IL-1 $\beta$ and recruitment of neutrophils in an experimental model of peritonitis and, similar to NLRP3- or ASC-deficient mice, are resistant to lipopolysaccharide-induced sepsis, which indicates a role for LUBAC in inflammasome-dependent inflammation.

J. Exp. Med. (23 June 2014) doi:10.1084/jem.20132486 